\title{
Shuffle Equations, Parallel Transition Systems and Equational Petri Nets
}

\author{
Stephen L. Bloom ${ }^{1}$ and Klaus Sutner \\ Stevens Institute of Technology \\ Hoboken, NJ 07030
}

\section{Motivation}

Consider how one might describe the sequences of atomic actions $[F]$ performable by a standard deterministic flowchart scheme $F$. One can exhibit $F$ as a finite directed graph whose vertices are labeled with letters in a ranked alphabet $\Omega$ (if the vertex $v$ has outdegree $k$, then the letter labeling $v$ is in the set $\Omega_{k}$ ). Then we say that the set $[F]$ is the set of all labels of paths in $F$ from the 'begin vertex' to the exit. If one wants to refine this notion further, one can associate a labeled tree with $F$, the so called 'unfolding' of $F$, and define $[F]$ as this tree. To describe the tree in great detail, one can show how to associate a tree of depth at most one with each node in the underlying graph of $F$. This association takes the form of a system of equations. For example, suppose that $F$ is the flowchart scheme:

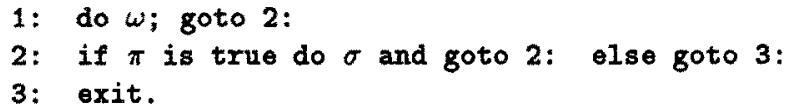

Then we can introduce a 'variable' $x_{i}$ for each instruction, and a corresponding equation (i):

$$
\begin{aligned}
& x_{1}=\omega \cdot x_{2} \\
& x_{2}=\pi_{t} \cdot \sigma \cdot x_{2}+\pi_{f} \cdot x_{3} \\
& x_{3}=\perp
\end{aligned}
$$

Then, one solves this system of equations in an appropriate structure.

Our work began with the question of describing the sequences of atomic actions performable by a flowchart algorithm which admits explicit nondeterminism and a forking type of parallelism. The kind of flowchart scheme is made precise below in our definition of a parallel transition system; the corresponding systems of equations are the shuffle equations. We then saw that the same equations were determined by a subclass of free choice Petri nets, which we call the equational Petri nets. Thus, shuffle equations, parallel transition systems and equational Petri nets are equivalent descriptions of this class of algorithm scheme. For reasons of space, we concentrate here on the equational Petri nets, and state only two results concerning the parallel transition systems.

\section{Preliminaries}

We let $N$ denote the set of nonnegative integers. For $n$ in $N,[n]$ is the set $\{1,2, \ldots, n\}$. A multiset on $X$ is a function $\nu: X \rightarrow N$; the collection of all multisets on $X$ will be denoted $N^{X}$. We identify a subset of $X$ with its characteristic function, so that, for example, the empty set is the multiset with

\footnotetext{
${ }^{1}$ Partially supported by NSF grant CCR-8901693
} 
constant value 0 . For any $x$ in $X, e_{x}: X \rightarrow N$ is the multiset with $e_{x}(y)=1$ if $y=x ; 0$ if $y \neq x$. If $\nu$ and $\nu^{\prime}$ are multisets, $\left(\nu+\nu^{\prime}\right)(y)=\nu(y)+\nu^{\prime}(y)$. We will also write $\nu-\nu^{\prime}$ for the function defined by pointwise subtraction, even if the value of this function is negative.

A word of length $n$ on a set $\mathrm{A}$ is a function $w:[n] \rightarrow A$, for some $n \geq 0$. An infinite word on $\mathrm{A}$ is a function $\{1,2, \ldots\} \rightarrow A$. We denote the unique word of length 0 by $\varepsilon$. The familiar operation of concatenation of words $w$ and $u$ is denoted $w \cdot u$. The set of words on $\mathbf{A}$ of finite length is $A^{*}$; the set of all finite and infinite words on $\mathrm{A}$ is $A^{\infty}$. For $Y \subset A^{\infty}$, we write pref $(\mathrm{Y})$ for the set of all finite words $u$ such that $u \cdot v \in Y$, for some $v \in A^{\infty}$. A ranked set $\Omega=\left\{\Omega_{k}: k=0,1, \ldots\right\}$ is a collection of pairwise disjoint sets $\Omega_{k}$. Elements in $\Omega_{k}$ are called function letters of rank $k$. For the remainder of the paper, $\Omega$ is a fixed ranked set such that $\Omega_{0}$ is the singleton set $\{\perp\}$. We will make use of the associated alphabet

$$
\Sigma:=\bigcup_{k \geq 1}\left(\Omega_{k} \times[k]\right)
$$

Thus, a letter in $\Sigma$ is of the form $(\omega, j)$ for some $\omega \in \Omega_{k}$ and some $j \in[k]$. A successor system $(V, \rho, \sigma)$ consists of a set $V$ (of vertices or nodes), a "rank" function $\rho: V \rightarrow N$, and functions $\sigma_{v}:[\rho(v)] \rightarrow V$, for $v$ in $V$; if $\sigma_{v}(i)=u$, we say that $u$ is the $i$-th successor of the vertex $v$. To avoid subscripts, we will sometimes write $\sigma(v, i)$ instead of $\sigma_{v}(i)$. A path in a successor system from $u$ to $v$ is a sequence $u=u_{0}, u_{1}, \ldots, u_{k}=v$ of vertices such that $u_{i+1}$ is a successor of $u_{i}$, for $i=0, \ldots, k-1$. A rooted successor system is a successor system having a distinguished vertex $r$, called the root. An $\Omega$-labeled successor system is a successor system equipped with a labeling function $\lambda$ from the vertices to $\Omega$ which respects the ranking - i.e. $\rho(v)=n$ iff $\lambda(v) \in \Omega_{n}$.

For later use, we define a binary operation \|. The operation is first defined as a map from pairs of finite words in $\Sigma^{*}$ to finite subsets of $\Sigma^{*}$.

$$
\begin{aligned}
u \| \varepsilon & :=\varepsilon \| u=\{u\}, \text { for all } u \in \Sigma^{*} \\
\sigma u \| \sigma^{\prime} v & :=\sigma \cdot\left(u \| \sigma^{\prime} v\right) \cup \sigma^{\prime} \cdot(\sigma u \| v) .
\end{aligned}
$$

If $U$ and $V$ are sets of finite words, $U \| V:=\bigcup\{u \| v: u \in U, v \in V\}$. If $\Omega$ is a ranked set, a $\Omega$ coalgebra $A$ consists of a set $A$ and a partial function

$$
\omega_{A}: A \rightarrow A \times[n]
$$

for each element $\omega$ in $\Omega_{n}, n \geq 1$. If $\omega \in \Omega_{0}, \omega_{A}$ is the empty function.

Example. The $\Omega$-coalgebra $U \Sigma$ has $\Sigma^{*}$ as its underlying set. The functions $\omega_{U \Sigma}$ are defined as follows. Let $s=\delta_{1} \delta_{2}, \ldots \delta_{n}$ be a word in $\Sigma^{*}$. If $\omega \in \Omega_{k}, k>0$, then

$$
\omega_{U \Sigma}:= \begin{cases}\left(\delta_{2} \ldots \delta_{n}, j\right) & \text { if } n>0 \text { and } \delta_{1}=(\omega, j) \\ \text { undefined } & \text { otherwise }\end{cases}
$$

\section{Parallel systems}

A parallel successor system is a rooted, $\Omega^{\sharp}$-labeled successor system $\Re$, where $\Omega^{\sharp}$ is obtained from $\Omega$ by the addition of the two symbols + and $\|$ to $\Omega_{2}$. If $\Re$ is a parallel system, an internal state of $\Re$ is an element of $N^{V} \times \Sigma^{*}$. Recall that $N^{V}$ is the collection of all multisets of vertices of $\Re$, and $\Sigma$ is the associated alphabet $\bigcup_{k} \geq 1\left(\Omega_{k} \times[k]\right)$. The meaning of the internal state $(\nu, s)$ is this: each vertex $v$ with $\nu(v)>0$ is allowed to "fire"; the word $s$ is a record of the sequence of "externally observable" actions which took place in the course of reaching the current internal state from the initial state. The second components of the internal states will be used to define the set of $\Re$-admissible words in $\Sigma *$ 
Definition $1 \Re$ determines a "transition relation", denoted $\Rightarrow \Re$, on the internal stales. We define the relation in two steps. First, for a vertex $v$ of $\Re$, we define $\Rightarrow_{\Re}^{v}$ as follows:

if $\nu(v)>0$ and one of the following conditions hold.

$$
(\nu, s) \Rightarrow\left(\nu^{\prime}, s^{\prime}\right)
$$

$1.1 \lambda(v)=+, s^{\prime}=s$ and

$\nu^{\prime}=\nu-e_{v}+e_{u}$, where $u$ is either $\sigma_{v}(1)$ or $\sigma_{v}(2)$.

$1.2 \lambda(v)=\|, s^{\prime}=s$ and

$\nu^{\prime}=\nu-e_{v}+e_{u}+e_{w}$, where $u=\sigma_{v}(1)$ and $w=\sigma_{v}(2)$.

1.3 the label of $v$ is $\omega \in \Omega_{k}, k>0$, and for some $i \in[k], s^{\prime}=s \cdot(\omega, i)$ and $\nu^{\prime}=\nu-e_{v}+e_{u}$, where $u=\sigma(v, i)$.

Lastly, we define

$1.4(\nu, s) \Rightarrow_{\Re}\left(\nu^{\prime}, s^{\prime}\right)$ if for some vertex $v,(\nu, s) \Rightarrow_{\Re}^{\nu}\left(\nu^{\prime}, s^{\prime}\right)$.

The relation defined by the first two conditions (1.1) and (1.2) only is called the "internal transition". Thus, we may say $(\nu, s) \Rightarrow\left(\nu^{\prime}, s\right)$ via an internal transition, meaning that either (1.1) or (1.2) holds. Similarly, if $(1.3)$ holds, we say $(\nu, s) \Rightarrow_{\Re}^{\vee}\left(\nu^{\prime}, s \cdot(\omega, j)\right)$ via a visible transition.

Definition 2 The set $\Sigma(\Re)$ of $\Re$-admissible words of a parallel system $\Re$ is defined as the set of all words $s$ in $\Sigma^{*}$ such that $\left(e_{r}, \varepsilon\right) \Rightarrow_{\Re}^{*}(\nu, s)$, for some $\nu$.

Let $\mathbf{A}=\left(A, \omega_{A}: \omega \in \Omega\right)$ be an $\Omega$-coalgebra. $\Re$ determines an $\mathbf{A}$-indexed collection $\widehat{\Re}_{A}$ of sets of finite and infinite sequences of elements in $A \times \mathrm{N}^{V} \times \Sigma^{*}$. In order to define which sequences belong to $\widehat{\Re}_{A}$, it is convenient to define three more binary relations.

Let $\Re$ be a fixed parallel system. Suppose that $\nu$ and $\nu^{\prime}$ are multisets of vertices of $\Re$, that $b$ and $c$ are elements of $\mathrm{A}$, and that $s$ and $s^{\prime}$ are words in $\Sigma^{*}$. Then

$$
(b, \nu, s) \vdash_{\Omega}^{v}\left(c, \nu^{\prime}, s^{\prime}\right)
$$

if $\nu(v)>0, \lambda(v)=\omega \in \Omega_{k}, k>0$, and for some $j$ in $[k], \omega_{A}(b)=(c, j), \sigma(v, j)=u, s^{\prime}=s \cdot(\omega, j)$ and $\nu^{\prime}=\nu-e_{v}+e_{u}$.

We write

if $(b, \nu, s) \vdash_{\Omega}^{v}\left(c, \nu^{\prime}, s^{\prime}\right)$, for some $v$.

$$
(b, \nu, s) \vdash_{\Omega}\left(c, \nu^{\prime}, s^{\prime}\right)
$$

$$
(b, \nu, s) \vdash_{\text {in }}\left(c, \nu^{\prime}, s^{\prime}\right)
$$

if $c=b, s^{\prime}=s$, and $(\nu, s) \Rightarrow \Re\left(\nu^{t}, s\right)$ via an internal transition (recall 1.1 and 1.2). Lastly, define

$$
(b, \nu, s) \vdash_{\Re, A}\left(c, \nu^{\prime}, s^{\prime}\right)
$$

if, for some multiset $\nu^{\prime \prime},(b, \nu, s) \vdash_{i n}^{*}\left(b, \nu^{\prime \prime}, s\right)$ and $\left(b, \nu^{\prime \prime}, s\right) \vdash_{\Omega}\left(c, \nu^{\prime}, s^{\prime}\right)$.

We note that $c=b$ and $s^{\prime}=s$ if $(b, \nu, s) \vdash_{\text {in }}^{*}\left(c, \nu^{\prime}, s^{\prime}\right)$. More importantly, if $(b, \nu, s) \vdash_{\Re_{i} A}\left(c, \nu^{\prime}, s^{\prime}\right)$, then $(\nu, s) \Rightarrow \Re\left(\nu^{\prime}, s^{\prime}\right)$.

Remark. Whenever there is a vertex $v$ in the current internal state which is labeled + or $\|$, then $\Re$ may change its internal state without changing the "external state", i.e. the $\mathbf{A}$-component. If $(b, \nu, s) \vdash_{i n}^{*}\left(b, \nu^{\prime}, s\right)$, then in state $(\nu, s), \Re$ is capable of making a sequence of invisible internal 
changes of its state. On the other hand, if $(b, \nu, s) \vdash_{\Omega}\left(c, \nu^{\prime}, s^{\prime}\right)$, then in state $(\nu, s)$, $\Re$ may make an external, visible change of state. The "names" of the observable changes of state which occurred are recorded in the word $s^{\prime}$.

Now we describe $\widehat{\Re}_{A}(a)$ for each element a in $A . \widehat{\Re}_{A}(a)$ is a set of sequences of elements in $A \times N^{V} \times \Sigma^{*}$. A finite or infinite sequence $\left(\xi_{0}, \xi_{1}, \ldots\right)$ belongs to $\Re_{A}(a)$ if firstly, $\xi_{0}=\left(a, e_{r}, \varepsilon\right)$ and $\xi_{n} \vdash_{\Re, A} \xi_{n+1}$ for each $n \geq 0$, whenever $\xi_{n}$ and $\xi_{n+1}$ are defined. Finally, $\widehat{\Re}_{A}$ is the function mapping $a \in A$ to the set of sequences $\widehat{\Re}_{A}(a)$.

For $\xi=(a, \nu, s)$ in $A \times N^{V} \times \Sigma^{*}$, write the $A$-component of $\xi$ as $p r_{A}(\xi)=a$. For $a$ in $A$, we define $\Re_{A}(a)$ as the set of all sequences $\left(p r_{A}\left(\xi_{1}\right), p r_{A}\left(\xi_{2}\right), \ldots\right)$ for $\left(\xi_{0}, \xi_{1}, \ldots\right)$ in $\widehat{\Re}_{A}(a)$. Note that we are deliberately omitting the first element $\xi_{0}$.

Definition 3 Let $\Re$ and $\Re^{\prime}$ be parallel systems. $\Re$ is trace equivalent to $\Re^{\prime}$ if for every coalgebra A, $\Re_{A}=\Re_{A}^{\prime}$, i.e. the sets of sequences $\Re_{A}(a)$ and $\Re_{A}(a)$ are identical, for each a in $A$.

A fundamental question concerning parallel systems is this. Is there an algorithm to determine, given two parallel systems $\Re$ and $\Re^{\prime}$, whether they are trace equivalent? We state a theorem which shows that this question reduces the the question of whether $\Re$ and $\Re^{\prime}$ determine the same sets of sequences on one particular coalgebra. Recall the definition of the coalgebra $U \Sigma$ in Example 2 of the previous section.

Theorem 4 Let $\Re$ and $\Re^{\prime}$ be parallel systems. The following statements are equivalent. $\Re$ is trace equivalent to $\Re^{\prime} . \Re_{U \Sigma}=\Re_{U \Sigma}^{\prime}, \Sigma(\Re)=\Sigma\left(\Re^{\prime}\right)$.

Our proof (omitted here) shows that the set $\Sigma(\Re)$ is determined by the following condition:

$s \in \Sigma(\Re) \Longleftrightarrow$ there is a sequence $f=\left(f_{1}, \ldots, f_{n}\right)$ in $\Re_{U \Sigma}(s)$ where $n$ is the length of $s$ and $f_{n}=\varepsilon$.

Equations. The last condition above shows the importance of the set $\Sigma(\Re)$ of $\Re$-admissible words. We can give another description of this set by means of a system of equations. Suppose that $\Re=(V, \rho, \sigma, r, \lambda)$ is a fixed finite parallel successor system. Choose an enumeration of $V$, say $V=\left\{v_{1}, \ldots, v_{m}\right\}$. We consider each vertex as a "variable". For each variable $v_{i}$ in $V$, we introduce a term $t_{i}$ as follows. If $\lambda\left(v_{i}\right)=\omega \in \Omega_{k}$, write $u_{j}$ for the $j-t h$ successor vertex of $v, \sigma(v, j)$, for each $j$ in $[k]$. Then the term $t_{i}$ is defined as

$$
t_{i}:=(\omega, 1) u_{1}+(\omega, 2) u_{2}+\ldots+(\omega, k) u_{k}
$$

if $\lambda\left(v_{i}\right)=\perp$, the term $t_{i}$ is just $\perp$. If $\lambda\left(v_{i}\right)=+$, then $t_{i}$ is $u_{1}+u_{2}$, where $u_{j}$ is the $j-t h$ successor of $v_{i}, j=1,2$. Lastly, if $\lambda\left(v_{i}\right)$ is $\|, t_{i}$ is $u_{1} \| u_{2}$, where again $u_{j}$ is the $j-t h$ successor of $v_{i} . \Re$ determines the system of fixed point equations

$$
v_{i}=t_{i}, i=1, \ldots, m \text {. }
$$

Conversely, such a system of equations in turn determines a parallel system. The labeling of the vertex $v_{i}$ can be deduced from the form of the corresponding term $t_{i}$, as can the values of the successor function. We take the root of the system to be $v_{1}$, the first variable. From now on, we identify a finite parallel system with a finite set of equations of the form 7 . One can solve this system in an algebra $C \Sigma$ of closed subsets of the finite and infinite words (on the alphabet of those letters that label the nodes of a parallel system), in which + is union and the operation $\|$ is the continuous extension of the operation given in Section 2, (5) and (6). 
Consider a finite system of equations,

$$
v_{i}=t_{i}, i=1, \ldots, m
$$

as above, where the set of variables on the left is $\left\{v_{1}, \ldots, v_{m}\right\}$. We will assume that the corresponding parallel system $\Re$ has root $v_{1}$ and that:

for each $\mathrm{i}$, there is a path in $\Re$ from $v_{1}$ to $v_{i}$;

for each $i$, there is a path in $\Re$ from $v_{i}$ to a vertex labeled $\omega \in \Omega_{k}$, for some $k>0$. every path of positive length from $v$ to itself contains at least one vertex labeled $\omega \in \Omega_{k}$, for some $k>0$.

Consider the $m$-tuple of terms $\tau:=\left\langle t_{1}\left(v_{1}, \ldots, v_{m}\right), \ldots, t_{m}\left(v_{1}, \ldots, v_{m}\right)\right\rangle$. If we substitute $t_{i}$ for $v_{i}$ simultaneously in each term $t_{1}, \ldots, t_{m}$, we get an $m$-tuple of terms $\tau^{2}$; after $m$ such substitutions, we get an $m$-tuple of terms $\tau^{m}=\left\langle h_{1}\left(v_{1}, \ldots, v_{m}\right), \ldots, h_{m}\left(v_{1}, \ldots, v_{m}\right)\right\rangle$. The meaning of the three conditions above is the following. Identifying each term $h_{i}$ with a finite tree in the usual way, every path from the root of $h_{i}$ to any leaf passes through some vertex labeled by a letter in $\Omega$. In this restricted situation, the set of equations (8) has a unique solution in $C \Sigma$, since the term $\tau^{m}$ is a proper contraction map.

Theorem 5 Let $\left(X_{1}, \ldots, X_{m}\right)$ be the unique solution of the system (8) of equations for the system $\Re$. Then, letting $\Re_{i}$ denote the parallel system with root $v_{i}, i=1, \ldots, m$, we have:

$$
\Sigma\left(\Re_{i}\right)=\operatorname{pref}\left(X_{i}\right) \text {. }
$$

In the next section, we will consider several decision problems connected with systems of equations of the form (8).

\section{Equational Petri Nets}

In the remaining sections we consider the class of languages $\Sigma(\Re)$ for parallel successor systems $\Re$. These languages also can be described in terms of a special class of Petri nets, which we call the equational Petri nets. We assume that the reader is familiar with the basic definitions concerning Petri nets and Petri net languages (see e.g. $[6,5,4,7,8]$ ). For our purposes, an equational Petri net is a quadruple $E=\langle P, T, \delta, \pi\rangle$ where $P$ is a finite set of places, $T$ is a finite set of transitions, and $\pi$ and $\delta$ are functions from the set of transitions to the set of places and multisets on $P$ respectively: $\pi: T \rightarrow P, \delta: T \rightarrow \mathbf{N}^{P}$.

The place $\pi(t)$ is called the source of the transition $t$. In contrast to general Petri nets, every transition is enabled by only one place, its source. A marking is a multiset on $P$. The support of a marking $m$ is the set $\{p \in \mathrm{P}: m(p)>0\}$. For two markings $m_{1}$ and $m_{2}$, we write $m_{1} \leq m_{2}$ if $\forall p \in P\left(m_{1}(p) \leq m_{2}(p)\right)$. The rank of the marking $m$ is the number of elements of $m$, i.e., $r k(m):=\Sigma_{p \in P} m(p)$. A marked equational Petri net is a pair $\left\langle E ; m_{0}\right)$ where $E$ is an equational Petri net and $m_{0}$ is a marking, called the initial marking.

We will abuse notation by identifying a place $p$ with the multiset $e_{p}$ introduced in section 1 . For a transition $t$ let $\Delta(t):=\delta(t)-\pi(t) \in \mathbf{Z}^{P}$ be the yield of $t$. A sequence of transitions is a firing sequence. For any marking $m$ and firing sequence $w \in T^{*}$, we define $m \cdot w \in \mathbf{Z}^{P}$ by induction on the length of $\mathrm{w}$ :

$$
m \cdot w:= \begin{cases}m & \text { if } w=\varepsilon \\ (m+\Delta(t)) \cdot u & \text { if } w=t \cdot u\end{cases}
$$


Definition $6 A$ transition $t$ is enabled at a marking $m$ if $m \geq \pi(t)$, i.e., $m(\pi(t))>0$. A firing sequence $w=t \cdot u, u \in T^{*}$ is enabled at $m$ if $t$ is enabled at $m$ and $u$ is enabled at $m \cdot t ; w \in T^{\infty}$ is enabled at $m$ if for each finite prefix $u$ of $w, u$ is enabled at $m$. (By definition, the empty sequence is enabled at every marking.)

We will use the following notation.

$$
\begin{aligned}
\mathcal{F}^{\infty}(m) & :=\left\{u \in T^{\infty} \mid u \text { enabled at } m\right\} \\
\mathcal{F}^{*}(m) & :=\left\{u \in T^{*} \mid u \text { enabled at } m\right\}, \\
\mathcal{R}(m) & :=\left\{m+\Delta(w) \mid w \in \mathcal{F}^{*}(m)\right\} .
\end{aligned}
$$

$\mathcal{R}(m)$ is the set of markings reachable from $m$.

For any set $\Gamma$, a $\Gamma$-labeled equational Petri net is a triple $\langle E ; \Gamma, h\rangle$ where $E$ is an EPN, and where $h$ is a function $h: T \rightarrow \Gamma$. We call $h$ a labeling of $T$ in $\Gamma$. In a $\Gamma \cup\{\varepsilon\}$-labeled net the labeling function has the format $h: T \rightarrow \Gamma \cup\{\varepsilon\}$. Similarly one defines labeled, marked EPNs. Define the finitary and infinitary exhaustive language of $E$ with marking $m_{0}$ by

$$
\begin{aligned}
\mathcal{L}^{*}\left(m_{0}\right) & :=h\left[\mathcal{F}^{*}\left(m_{0}\right)\right] \subset \Gamma^{*} \\
\mathcal{L}^{\infty}\left(m_{0}\right) & :=h\left[\mathcal{F}^{\infty}\left(m_{0}\right)\right] \subset \Gamma^{\infty}
\end{aligned}
$$

For simplicity we will frequently write just $\left|m_{0}\right|$ for $\mathcal{L}^{*}\left(m_{0}\right)$ and $\left|m_{0}\right|_{T}$ for $\mathcal{F}^{*}\left(m_{0}\right)$. If the underlying net is not clear from context we will also write $\mathcal{F}^{\infty}\left(m_{0} ; E\right), \mathcal{L}^{*}\left(m_{0} ; E\right)$ and so forth.

A language $L \subset \Gamma^{*}$ is an equational Petri net language (EPNL for short) iff for some $\Gamma$-labeled equational Petri net $E$ and some marking $m_{0}: L=\mathcal{L}^{*}\left(m_{0} ; E\right)$. Example 1 shows that EPNLs in general fail to be context free, even if the corresponding net is very simple.

It will be convenient to assume that $\delta(t)>0$ for all transitions $t$ in an equational Petri net. This property can be achieved by adding another place $p_{g}$ to the net if necessary (for "garbage collection") and if $\delta(t)=0$ for some transition $t$, we redefine $\delta$ at $t$ by: $\delta(t)=p_{g}$ (see example 1). Furthermore, we will assume that there is at most one place that does not occur as the source of any transition. It is clear from the definitions just given that these conventions do not affect the firing sequences that are enabled in the net.

Example 1

For any alphabet $\Gamma$, a symbol $\gamma \in \Gamma$ let $\#_{\gamma}: \Gamma^{*} \rightarrow \mathbf{N}$ be the Parikh map: \# ${ }_{\gamma}(x)$ is the number of occurrences of symbol $\gamma$ in word $x \in \Gamma^{*}$. Define a net $E_{0}$ by $P=\left\{p_{0}, p_{1}, p_{2}, p_{g}\right\}, T=\left\{t_{1}, t_{2}, t_{3}\right\}$, $h\left(t_{1}\right)=a, h\left(t_{2}\right)=b, h\left(t_{3}\right)=c, \pi\left(t_{i}\right)=p_{i}$ and $\delta\left(t_{1}\right)=p_{0}+p_{1}+p_{2}, \delta\left(t_{2}\right)=\delta\left(t_{3}\right)=p_{g}$. Let $m_{0}:=p_{0}$ be the initial marking. Then $\left|m_{0}\right|=\left\{z \in\{a, b, c\}^{*} \mid \forall x \in \operatorname{Pref}(z)\left(\#_{a} x \geq \#_{b} x\right.\right.$, \#c $\left.\left.x\right)\right\}$. See figure 1 for a graphical representation of this net.

\section{$5 \quad$ Equivalence of EPNLs and Languages of Admissible Words}

There is a natural way to associate to a parallel successor system $\Re=\langle V, \rho, \sigma\rangle$ an $\Sigma \cup\{\varepsilon\}$-labeled equational Petri net $E^{\prime}$. The net has as places the vertices of $\Re$ and its transitions are defined as follows. If the label of $v$ is $\omega \in \Sigma_{k}, k>0$, then there are $k$ transitions $t_{i}$ in the net labeled $(\omega, i), i \in[k]$, such that $\pi\left(t_{i}\right)=v$ and $\delta\left(t_{i}\right)=\sigma_{v}(i)$. If there is a vertex $v$ in $\Re$ labeled $\perp$ then the corresponding place has outdegree 0 . If the label of $v$ is t then there are transitions $t_{i}, i=1,2$, labeled $\varepsilon$ having source $v$ such that $\delta\left(t_{i}\right)=\sigma_{v}(i), i=1,2$. Lastly, if the label of $v$ is ' $\|$ ' then there is a transition $t$ with source $v$ labeled $\varepsilon$ such that $\delta(t)$ is the multiset $\sigma_{v}(1)+\sigma_{v}(2)$. With these definitions it is quite straightforward to show that $\Sigma(\Re)=\mathcal{L}\left(r ; E_{\Re}^{\prime}\right)$, where $r$ is the root of $\Re$. 


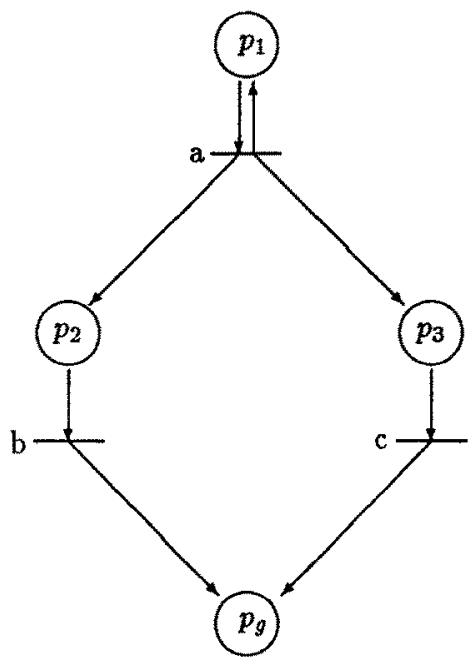

Figure 1: A primitive equational Petri net.

For a place $p$ and a symbol $\sigma \in \Sigma$ define eps $(p, \omega):=\left\{m \in N^{P} \mid p \cdot w=m \wedge h(w)=\omega\right\}$. Thus $e p s(p, \omega)$ is the set of all markings that can be reached from $p$ by firing a transition labeled $\omega$ plus any number of $\varepsilon$-labeled transitions. If the parallel system satisfies condition 3 , Section 2 , then $e p s(p, \omega)$ must be finite for all places $p$ in $E_{\Re}^{\prime}$. This is the key to eliminating $\varepsilon$-labeled transitions from the net. Briefly, a new $\Gamma$-labeled net $E_{\Re}$ is obtained from $E_{\Re}^{\prime}$ by deleting all the old transitions and, for every $p$ in $P$ and every marking $m$ in $e p s(p, \omega)$, introducing a new transition $t$ labeled $\omega$ such that $\delta(t)=(p, m)$. Furthermore, one new place $q$ with transitions $t$ such that $h(t)=\omega$ and $\delta(t)=(q, m)$ for all $m \in \operatorname{eps}(r, \omega)$ is added to the net. Here $r \in V=P$ is the root of the successor system. Then an easy induction shows that $\mathcal{L}\left(q ; E_{\Re}\right)=\Sigma(\Re)$.

It is somewhat easier to describe the system of equations determined by a $\Gamma$-labeled EPN $E$; the variables appearing in the equations are the places, and for each place $x$ and each transition $t$ with $\pi(t)=x$ we let the term $\tau(x, t)$ be defined by

$$
\tau(x, t):=\omega\left(y_{1}\left\|y_{2}\right\| \cdots \| y_{k}\right)
$$

where the label of $t$ is $\omega$ and $\delta(t)=y_{1}+\cdots+y_{k}$ (there may be repetitions among the $y$ 's). The equation corresponding to the place $x$ is then

$$
x=\tau\left(x, t_{1}\right)+\cdots+\tau\left(x, t_{m}\right)
$$

where $t_{1}, \ldots, t_{m}$ are all transitions with source $x$. (If $x$ is a place of outdegree 0 , the last equation becomes $x=\perp$, or, equivalently in the algebra $C \Sigma, x=\{\varepsilon\}$.) The system of equations determined by $E$ is the set of such equations, one for each place $x$ in $E$. Note that these equations are already in normal form.

\section{Example 2}

Consider a parallel successor system $\Re$ defined by $V=\left\{r, v_{1}, v_{2}, u_{1}, u_{2}\right\}, \rho(r)=1, \rho\left(v_{i}\right)=2, \rho\left(u_{i}\right)=0$ and lastly $\sigma_{r}(1)=v_{1}, \sigma_{v_{1}}(1)=r, \sigma_{v_{1}}(2)=v_{2}$ and $\sigma_{v_{2}}(i)=u_{i}$. The node-labels are given by $\lambda(r)=a$, $\lambda\left(u_{1}\right)=b$ and $\lambda\left(u_{2}\right)=c,\{a, b, c\}=\Omega_{1}, \lambda\left(u_{3}\right)=\perp \in \Omega_{0} ; \lambda\left(v_{i}\right)=\|, i=1,2$. The corresponding system of equations is 


$$
\begin{aligned}
& v_{1}=\|\left(r, v_{2}\right), \\
& v_{2}=\|\left(u_{1}, u_{2}\right), \\
& u_{1}=b \cdot u_{3}, \\
& u_{2}=c \cdot u_{3}, \\
& u_{3}=\perp .
\end{aligned}
$$

Now define an equational Petri net $E_{\Re^{\prime}}$ as follows. Let $P=V$ and $T=\left\{t_{i} \mid i \in[5]\right\}$. The maps $\pi$ and $\delta$ as well as labeling $h$ are defined in the following table.

Performing the $\varepsilon$-elimination procedure described above yields the following net $E_{\mathfrak{1}}$. There are 13 transitions $t_{1}, \ldots, t_{13}$; the functions $\pi$ and $\delta$ as well as labeling $h$ are again given by a table as follows. Note that $\mathcal{L}\left(r ; E_{\Re}\right)=\mathcal{L}\left(m_{0} ; E_{0}\right)$ where $E_{0}$ is the net from example 1 .

\begin{tabular}{|c|c|c|l|}
\hline$i$ & $h\left(t_{i}\right)$ & $\pi\left(t_{i}\right)$ & $\delta\left(t_{i}\right)$ \\
\hline 1 & $a$ & $r$ & $v_{1}$ \\
2 & $\varepsilon$ & $v_{1}$ & $r+v_{2}$ \\
3 & $\varepsilon$ & $v_{2}$ & $u_{1}+u_{2}$ \\
4 & $b$ & $u_{1}$ & $u_{3}$ \\
5 & $c$ & $u_{2}$ & $u_{3}$ \\
\hline
\end{tabular}

\begin{tabular}{|c|c|c|l|}
\hline$i$ & $h\left(t_{i}\right)$ & $\pi\left(t_{i}\right)$ & $\delta\left(t_{i}\right)$ \\
\hline 1 & $a$ & $r$ & $v_{1}$ \\
2 & $a$ & $r$ & $r+v_{2}$ \\
3 & $a$ & $r$ & $r+u_{1}+u_{2}$ \\
4 & $a$ & $v_{1}$ & $v_{1}+v_{2}$ \\
5 & $a$ & $v_{1}$ & $r+2 v_{2}$ \\
6 & $a$ & $v_{1}$ & $r+v_{2}+u_{1}+u_{2}$ \\
7 & $a$ & $v_{1}$ & $r+2 u_{1}+2 u_{2}$ \\
8 & $b$ & $v_{1}$ & $r+u_{2}+u_{3}$ \\
9 & $c$ & $v_{1}$ & $r+u_{1}+u_{3}$ \\
10 & $b$ & $v_{2}$ & $u_{2}+u_{3}$ \\
11 & $c$ & $v_{2}$ & $u_{1}+u_{3}$ \\
12 & $b$ & $u_{1}$ & $u_{3}$ \\
13 & $c$ & $u_{2}$ & $u_{3}$ \\
\hline
\end{tabular}

The next three propositions indicate some major differences between general Petri nets and equational Petri nets. We omit their proofs. Let $\Gamma=\left\{\omega_{1}, \ldots, \omega_{k}\right\}$ and define the full Parikh map \# : $\Gamma^{*} \rightarrow N^{k}$ by $\#(x):=\left(\# \omega_{\omega_{1}} x, \ldots, \# \omega_{\omega_{k}} x\right)$.

Proposition 7 In any $\Gamma$-labeled equational Petri net $E$ with markings $m_{1}, m_{2}$ we have: $\left|m_{1}+m_{2}\right|=$ $\left|m_{1}\right| \|\left|m_{2}\right|$.

It is demonstrated in [1] that the problem of testing equality of sets of reachable markings is undecidable for general Petri nets. However, this problem is decidable for equational Petri nets due to the simple periodic structure of their set of reachable markings.

Proposition 8 In any equational Petri net, the set of reachable markings $\mathcal{R}(m)$ is semi-linear. Thus it is decidable whether two marked equational Petri nets have the same set of reachable markings.

A similar argument shows that the commutative image of a EPNL is semi-linear. Hence many EPNLs are examples of non context free languages with semi-linear commutative image.

Proposition 9 For every equational Petri net language $L$, the commutative image \# $(L)$ is semilinear. Thus it is decidable if two equational Petri net languages are letter-equivalent. 


\section{Regular and Context Free EPNLs}

Clearly every Petri net language is context sensitive. As example 1 shows, even primitive equational Petri nets may generate languages that fail to be context free. We will now give necessary and sufficient conditions for the set of firing sequences of an equational Petri net to be regular or context free, respectively. A characterization of those Petri nets generating a regular exhaustive language can be found in [9].

\subsection{Firing Trees}

The linear order inherent in a firing sequence obscures the concurrent nature of a Petri net. Therefore we introduce the notion of a firing tree: a firing tree is essentially a derivation tree in an appropriate rewriting system. Let $E$ be an equational Petri net, $m$ a marking and suppose $w \in|m|_{T}$. Assume some fixed linear ordering of the places in $E$. Define a forest $T R(m, w)$ consisting of exactly $r k(m)$ ordered trees whose nodes are labeled in $P . T R(m, w)$ is defined by induction on $w$ as follows. $-T R(m, \varepsilon)$ consists of $r k(m)$ isolated roots, the label of the i-th root is $p$ where $\Sigma_{q<p} m(q)<i \leq \Sigma_{q \leq p} m(q)$. $T R(m, w t)$ is obtained from $T R(m, w)$ by finding the leftmost occurrence of a leaf $z$ labeled $p=\pi(t)$ and attaching new leaves $z_{1}, \ldots, z_{k}$ to $z$ where $k=r k(\delta(t))$. The label of $z_{i}$ is $q$ where $\Sigma_{q^{\prime}<q} \delta(t)\left(q^{\prime}\right)<$ $\left.i \leq \Sigma_{q^{\prime}} \leq q\right)(t)\left(q^{\prime}\right)$. We will write $\lambda(z)$ for this label. Now let $w$ be a firing sequence $w$ enabled at $m$ and let $p$ be a place. It is obvious from the definitions that

- the length of $w$ is the number of interior nodes in $T R(m, w)$ and

$-(m \cdot w)(p)$ is the number of leaves in $T R(m, w)$ labeled $p$.

A place $p$ in $E$ is proper if there is a transition $t$ enabled at $p$ such that $\Delta(t)(p)=-1$, improper otherwise. A set $Q$ of places is unbounded (for the marking $m_{0}$ ) if $\forall k \geq 0 \exists m \in \mathcal{R}\left(m_{0}\right) \forall p \in$ $Q(m(p) \geq k)$. A set $Q$ of places is proper unbounded iff $Q$ is unbounded and all the places in $Q$ are proper. Lastly a net is $k$-unbounded iff $k=\max (|Q| \mid Q \subset P$ proper unbounded).

\subsection{The Collapse of an Equational Petri Net}

In what follows it will be convenient to think of an equational Petri net $E$ as a bi-partite multidigraph. Thus for each transition $t$ with source $p$ there is an edge from $p$ to $t$ and edges from $t$ to $q$ (with multiplicity $\delta(t)(q)$ ) for all $q$ in the support of $\delta(t)$. Note that the indegree of every vertex $t$ is at most one. The net $E$ is primitive iff the corresponding graph contains no strongly connected components (scc, for short) with more that two vertices. We now associate every equational Petri net $E$ with a primitive net $\mathcal{C}(E)$, the collapse of $E$. The nets $E$ and $\mathcal{C}(E)$ will have essentially the same sets of unbounded places. $\mathcal{C}(E)$ is obtained from $E$ by collapsing all the strongly connected components $C$ of $E$ of size at least two into one place $p_{C}$ and one transition $t_{C}$.

We will distinguish several types of strongly connected components as follows. Let $C$ be a strongly connected component of $E$ of size at least two.

$C$ is inactive if $\forall t \in C$ (outdegree $(t) \leq 1)$; otherwise $C$ is active. More specifically, $C$ is externally active if $\forall t \in C$ (outdegree $C(t) \leq 1) \wedge \exists t \in C$ (outdegree $(t) \geq 2$ ). $C$ is internally active if $\exists t \in$ $C$ (outdegree $C(t) \geq 2$ ).

Lastly define a collapse map $\kappa$ from $E$ onto $\mathcal{C}(E)$ by

$$
\kappa(z):= \begin{cases}z & \text { if the } \operatorname{scc} C \text { of } z \text { is }\{z\} \\ z_{C} & \text { otherwise. }\end{cases}
$$

Note that $\mathcal{C}(E)$ can be constructed from $E$ in polynomial time using standard graph theory algorithms. 
The net $E_{0}$ from example 1 is primitive: it has only one non-trivial scc, namely $\left\{p_{0}, t_{1}\right\}$. This component is externally active. The place $p_{0}$ is improper, and $\left\{p_{1}, p_{2}, p_{g}\right\}$ is a proper unbounded set of places. As we will see shortly this implies that the language of the net is not context free. The net $E_{\Re}$ from example 2 also has only one active component, namely $\left\{r, v_{1}, t_{1}, \ldots, t_{7}\right\}$. All places are proper, and the set $\left\{v_{2}, u_{1}, u_{2}, u_{3}, u_{3}\right\}$ is proper unbounded.

Lemma 10 Let $E$ be a marked equational Petri net, $\mathcal{C}(E)$ its collapse. Then a set $Q$ of places is unbounded in $E$ iff $\kappa[Q]$ is unbounded in $\mathcal{C}(E)$.

Proof. It clearly suffices to verify the following two claims. For all firing sequences $w$ in $E$ enabled at $m_{0}$ there exists a firing sequence $w^{\prime}$ in $C(E)$ such that

$$
\kappa\left(m_{0} \cdot w\right) \leq \kappa\left(m_{0}\right) \cdot w^{\prime}
$$

Conversely, for all firing sequences $w^{\prime}$ in $\mathcal{C}(E)$ enabled at $\kappa\left(m_{0}\right)$ there exists a firing sequence $w$ in $E$ such that

$$
\kappa\left(m_{0}\right) \cdot w^{\prime} \leq \kappa\left(m_{0} \cdot w\right) .
$$

Since the proofs are similar, we give only the second argument. We proceed by induction on $w^{\prime}$. Suppose $w^{\prime}=u^{\prime} \cdot t^{\prime}$ where $t^{\prime}$ is some transition in $\mathcal{C}(E)$. By induction hypothesis $\kappa\left(m_{0}\right) \cdot u^{\prime} \leq \kappa\left(m_{0} \cdot u\right)$ for some firing sequence $u$ in $E$. If $t^{\prime}$ is one of the transitions unaffected by the collapse (because the corresponding strongly connected component contains only $t^{\prime}$ ), then we may set $w:=u \cdot t^{\prime}$. So assume $t^{\prime}=t_{C}$. Note that at least one of the transitions in $C$ is enabled at $m_{0} \cdot u$. We distinguish several cases depending on the type of the strongly connected component $C$.

Case $1 C$ is inactive.

In this case $\Delta\left(t_{C}\right)=0$. Pick a transition $t$ in $C$ which is enabled at $m_{0} \cdot u$ and set $w:=u \cdot t$. Note that $\kappa\left(m_{0}\right) \cdot u^{\prime} \cdot t_{C}=\kappa\left(m_{0} \cdot u^{\prime}\right) \leq \kappa\left(m_{0} \cdot u\right)=\kappa\left(m_{0} \cdot u \cdot t\right)$.

Case $2 C$ is active.

In this case $\Delta\left(t_{C}\right)>0$ and possibly $\Delta\left(t_{C}\right)\left(p_{C}\right)=1$ (if $C$ is internally active). Pick a firing sequence $v$ in $C$ enabled at $m_{0} \cdot u$ such that $\kappa(\Delta(v)) \geq \Delta\left(t_{C}\right)$. The existence of such a sequence follows from the fact that $C$ is strongly connected and the definition of $\mathcal{C}(E)$ : any edge $\left(t_{C}, z\right)$ in $\mathcal{C}(E)$ comes from an edge $(t, q)$ where $t$ is a transition in $C$. Now set $w:=u \cdot v$. Note that $\kappa\left(m_{0}\right) \cdot u^{\prime} \cdot t_{C}=$ $\kappa\left(m_{0} \cdot u^{\prime}\right)+\Delta\left(t_{C}\right) \leq \kappa\left(m_{0} \cdot u\right)+\kappa(\Delta(v))=\kappa\left(m_{0} \cdot u \cdot v\right)$.

The following lemma establishes a normal form for the firing sequences that show that a set of places is unbounded.

Lemma 11 Suppose that $E$ is a primitive equational Petri net, $Q=\left\{q_{1}, \ldots, q_{n}\right\}$ a set of places in $E$ and that $m_{0}$ is a marking. Then $Q$ is unbounded for $m_{0}$ iff there exist repetition-free firing sequences $u_{i}, v_{i}$ and transitions $t_{i}, i=1, \ldots, n$, such that

$$
\forall k \geq 0, q \in Q\left(m_{0} \cdot u_{1} t_{1}^{k} v_{1}^{k} \ldots u_{n} t_{n}^{k} v_{n}^{k}(q) \geq k\right) .
$$

Proof. The sufficiency of our condition is obvious, to see necessity let $T_{0} \subset T$ be the set of self-loop transitions in $E$ with positive yield, and let $P_{0}$ be the corresponding set of places. Suppose $Q$ is unbounded for $m_{0}$. Then for any $k \geq 0$ there must be a firing sequence $w$ enabled at $m_{0}$ such that the firing tree $T R\left(m_{0}, w\right)$ contains at least $k$ leaves labeled $q$ for all $q$ in $Q$. Choose $k:=r k\left(m_{0}\right) \cdot r^{2|T|}$ where $r:=\max (\delta(t) \mid t \in T)$ and let $w$ be a minimal such firing sequence. Then at least one of the trees in $T R\left(m_{0}, w\right)$ must contain one of the transitions in $T_{0}$. Suppose $\delta(t)=\pi(p)+d$ where $d>0$ is the top-most occurrence of any such transition. Then there is a repetition-free sequence $u_{1}$ of transitions in $T-T_{0}$ such that $u_{1} t^{*} \subset\left|m_{0}\right| T$. Let $\left\{q_{1}, \ldots, q_{j}\right\} \subset Q$ be all the places in $Q$ that occur in at least one of the $r k(d)$ subtrees associated with the transition $t$. By the minimality of $w$, we have $j \geq 1$. 
Hence there exist repetition-free firing sequences $v_{1}, \ldots, v_{j}$ such that $m_{0} \cdot u_{1} t_{1}^{k} v_{1}^{k} \ldots t_{1}^{k} v_{j}^{k}\left(q_{i}\right) \geq k$ for $i=1, \ldots, j$. Now delete all subtrees from $T R\left(m_{0}, w\right)$ that contain no leaves in $Q_{1}:=Q-\left\{q_{1}, \ldots, q_{j}\right\}$. Replace $m_{0}$ by $m_{1}:=m_{0} \cdot v_{1}$ and $Q$ by $Q_{1}$ and proceed by induction.

Note that in general an equational Petri net has an exponential number of maximal unbounded sets of places. Hence we cannot hope to enumerate them in polynomial time. However, the next theorem shows that it is already NP-complete to determine whether a single given set $Q$ of places is unbounded. More precisely, consider the following decision problem.

\section{Problem: Boundedness for EPNs}

Instance: An equational Petri net $E$, a marking $m$ and a set $Q$ of places in $E$. Question: Is $Q$ an unbounded set of places for $m$ in $E$ ?

Remark. The reachability problem for EPN's has been shown to be NP-complete in [3]. A related boundedness problem has been considered in [2].

Theorem 12 The problem of Boundedness for EPNs is NP-complete. The problem remains NP. complete even if the net is required to be primitive.

Proof. To see membership in NP first note that the primitive net $\mathcal{C}(E)$, the collapse of an equational Petri net $E$, can be computed in polynomial time. Hence, by lemma 10 , we may safely assume that we are dealing with a primitive net. So let $Q=\left\{q_{1}, \ldots, q_{n}\right\}$ a set of places in $E$ and $m_{0}$ a marking. By lemma 11 , the set $Q$ is unbounded for $m_{0}$ iff there exist repetition-free firing sequences $u_{i}, v_{i}$ and transitions $t_{i}, \quad i=1, \ldots, n$, such that $\forall k \geq 0, q \in Q\left(m_{0} \cdot u_{1} t_{1}^{k} v_{1}^{k} \ldots u_{n} t_{n}^{k} v_{n}^{k}(q) \geq k\right)$. The total size of $u_{1}, \ldots, u_{n}, v_{1}, \ldots, v_{n}$ and $t_{1}, \ldots, t_{n}$ is polynomial in the size of $E$. Hence one can guess in non-deterministic polynomial time at these firing sequences and verify in deterministic polynomial time that they indeed have the desired properties.

To show hardness we will embed 3-Satisfiability (3SAT). An instance of 3SAT is a Boolean formula $\Phi=\phi_{1} \wedge \phi_{2} \wedge \ldots \wedge \phi_{m}$ in 3-conjunctive normal form using variables in $X=\left\{x_{1}, \ldots, x_{n}\right\}$. Suppose clause $\phi_{i}$ is $z_{i, 1} \vee z_{i, 2} \vee z_{i, 3}$ where the $z_{i, j}$ are literals over $X$. Define a primitive equational Petri net $E$ as follows. $E$ has places $p_{i, j}, \quad i=1, \ldots, n, j=0,1,2$ and $q_{k}, k=1, \ldots, m$, the 'pockets'. There is a transition $t_{i, j}$ with yield $p_{i, j}-p_{i, 0}, j=1,2, i \in[n]$. Furthermore, there is a transition $s_{i, 1}$

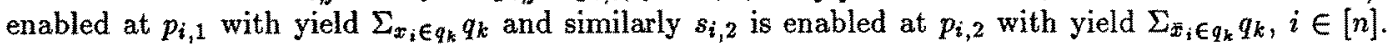
Hence the net consists of $n$ basic components corresponding to the $n$ boolean variables in $\Phi$ plus $m$ pockets corresponding to the clauses in $\Phi$. Now consider the initial marking $m_{0}:=p_{1,0}+\cdots+p_{n, 0}$. In each component the first transition fired determines whether the corresponding variable is true or false. The subsequent firings then allow the creation of an unbounded marking in those pockets whose corresponding clauses are satisfied by that particular choice of an assignment. It is easy to verify that $\Phi$ is satisfiable iff $Q:=\left\{q_{1}, \ldots, q_{m}\right\}$ is unbounded for the initial marking $m_{0}$.

In contrast to the computational hardness of unboundedness for sets of places of arbitrary size we will show that for a set $Q$ of cardinality at most 2 one can determine in polynomial time whether $Q$ is unbounded. Hence there is a polynomial time algorithm to decide whether a net is 0 -unbounded or 1-unbounded.

For a strongly connected component $C$ of an equational Petri net $E$ let $D(C):=\left\{p \mid \exists t, q_{1}, q_{2} \in\right.$ $\left.C\left(\pi(t)=q_{1} \wedge \delta(t)\left(q_{2}\right)>0 \wedge \delta(t)(p)>0\right)\right\} . D(C)$ is the collection of all places that can be "pumped" directly via firing sequences entirely within $C$. Clearly $D(C)$ can be determined in polynomial time. As a consequence of lemma 10 and lemma 11 we have the following two corollaries.

Lemma 13 Let $E$ be an equational Petri net, $m_{0}$ a marking and $Q=\{q\}$. Then $Q$ is unbounded iff there exists an active strongly connected component $C$ reachable from the support of $m_{0}$ such that $q$ is reachable from $D(C)$. 
Corollary 14 Let $E$ be an equational Petri net, $m_{0}$ a marking and $Q=\left\{q_{1}, q_{2}\right\}$. Then $Q$ is unbounded iff there exist two active strongly connected components $C_{1}$ and $C_{2}$ (not necessarily distinct) reachable from $p_{1}$ and $p_{2}$ respectively such that $q_{i}$ is reachable from $D\left(C_{i}\right), i=1,2$, and one of the following three conditions holds: $m_{0} \geq p_{1}+p_{2}, m_{0} \geq p_{1}$ and $p_{2} \in C_{1}$, or $m_{0} \geq p_{2}$ and $p_{1} \in C_{2}$.

Theorem 15 The set of all firing sequences enabled at $m_{0}$ in an equational Petri net $E$ is regular iff $E$ is 0 -unbounded, i.e., $E$ contains no proper unbounded places with respect to $m_{0}$.

Proof. First suppose $E$ is 0 -unbounded. Let $P_{0}$ be the collection of all proper places in $E$. Then for some constant $c$ we have: $\forall m \in \mathcal{R}\left(m_{0}\right), p \in P_{0}(m(p) \leq c)$. But for any improper place $p, m(p)>0$ and $m^{\prime} \in \mathcal{R}(m)$ implies $m^{\prime}(p)>0$; firing any transition enabled at $p$ does not decrease $m(p)$. Now define the marking $m^{\dagger}$ by

$$
m^{\dagger}(p):= \begin{cases}m(p) & \text { if } p \text { proper } \\ \min (m(p), 1) & \text { otherwise. }\end{cases}
$$

Define a finite sink automaton $M$ as follows: $M$ has states $\{0, \ldots, c\}^{P_{0}} \times\{0,1\}^{P_{0}} \cup\{\perp\}$, where $m_{0}^{\dagger}$ is the initial state and $\perp$ is the sink. The transition function is defined by

$$
\delta(m, t):= \begin{cases}(m \cdot t)^{\dagger} & \text { if } t \text { is enabled at } m \\ \perp & \text { otherwise. }\end{cases}
$$

Clearly $M$ accepts a firing sequence $w$ iff $w$ is enabled at $m_{0}$. For the opposite direction assume that $E$ contains at least one proper unbounded place $p_{0}$. By lemma 11 there exist firing sequences $u$ and $w$ such that $\forall k \geq 0\left(\left(m \cdot u w^{k}\right)\left(p_{0}\right) \geq k\right)$. Let $t$ be a transition enabled at $p$ such that $\Delta(t)(p)=-1$. Suppose for the sake of a contradiction that $\left|m_{0}\right| x$ is regular. Note that for all $k \geq 0 ; u w^{k} t^{k}$ is enabled at $m_{0}$. By the pumping lemma there is some $r$ sufficiently large such that $u w^{r} t^{*} \subset\left|m_{0}\right|$, contradiction.

Theorem 16 The set of all firing sequences enabled at $m_{0}$ in an equational Petri net $E$ is context free (but not regular) iff $E$ is 1 -unbounded, i.e., any set of proper unbounded places with respect to $m_{0}$ has cardinality at most one and there exists at least one such set.

Proof. First suppose $E$ is 1-unbounded. Again let $P_{0}$ be the collection of all proper places in $E$. For any $c \geq 0$ call a marking $m$ c-critical iff $\exists p(m(p) \geq c)$. As an immediate consequence of the 1-unboundedness of $E$ we have for some sufficiently large $c_{0}$ and all $m$ in $\mathcal{R}\left(m_{0}\right)$ there exists at most one proper place $p$ such that $m(p) \geq c_{0}$. Thus for all $c \geq c_{0}$ and $m$ in $\mathcal{R}\left(m_{0}\right)$ c-critical let $c r i t(m, c)$ denote that uniquely determined place.

Claim There exists a constant $c_{1} \geq c_{0}$ such that for all $m \in \mathcal{R}\left(m_{0}\right)$ and $m^{\prime} \in \mathcal{R}(m) c_{0}$-critical we have: $\operatorname{crit}\left(m, c_{1}\right)=\operatorname{crit}\left(m^{\prime}, c_{1}\right)$. For suppose otherwise. Then there are proper places $p \neq q$ such that for any $k$ we have $m_{k}$ in $\mathcal{R}\left(m_{0}\right)$ and $m_{k}^{\prime}$ in $\mathcal{R}\left(m_{k}\right)$ such that $m_{k}(p) \geq k$ and $m_{k}^{\prime}(q) \geq k$. But then for any $r \geq 0$ there are infinitely many pairs $i<j$ such that $m_{i}+r p \leq m_{j}$. Note that $m_{i}^{\prime}+r p$ is in $\mathcal{R}\left(m_{j}\right)$. But then $\{p, q\}$ is unbounded: $\left(m_{i}^{\prime}+r p\right)(p) \geq r$ and $\left(m_{i}^{\prime}+r p\right)(q) \geq i$, contradiction.

Now we can define a counter automaton $M$ that simulates the equational Petri net $E$ as follows. Let $c_{1}$ be as in the claim. For any marking $m$ define $m^{\dagger}$ by

$$
m^{\dagger}(p):= \begin{cases}m(p) & \text { if } m(p)<c_{1}, p \text { proper } \\ \min (m(p), 1) & \text { if } p \text { improper } \\ c_{1} & \text { if } p=\operatorname{crit}\left(m, c_{1}\right)\end{cases}
$$

$M$ has states $\{0, \ldots, c\}^{P} \cup\{1\}$, initial state $m_{0}^{\dagger}$ and every state other than $\perp$ is final. $M$ works as follows: as long as the marking $m$ of the net is non-critical $m$ is represented by state $m^{\dagger}$ of $M$ and the 
stack is empty. When the marking becomes $c_{1}$-critical for the first time $M$ switches mode: from now on it uses its stack to represent $m(p), p:=\operatorname{crit}\left(m, c_{1}\right)$, all other places are represented by the state $m^{\dagger}$. By the claim all markings reachable in the future are not $c_{1}$-critical in any component other than $p$. Hence $M$ properly simulates $E$. We leave the details to the reader.

On the other hand assume that $\left\{p_{1}, p_{2}\right\}$ is proper unbounded, $p_{1} \neq p_{2}$. By lemma 11 there exist firing sequences $u_{1}, u_{2}, w_{1}$ and $w_{2}$ such that $\forall k \geq 0\left(\left(m_{0} \cdot u_{1} w_{1}^{k} u_{2} w_{2}^{k}\right)\left(p_{i}\right) \geq k\right), i=1,2$. Let $t_{i}$ be a transition enabled at $p_{i}$ respectively such that $\Delta\left(t_{i}\right)\left(p_{i}\right)=-1$. As in the last argument for all $k \geq 0$ the firing sequence $u_{1} w_{1}^{k} u_{2} w_{2}^{k} t_{1}^{k} t_{2}^{k}$ is enabled at $m_{0}$. By the iteration theorem for context free languages $u_{1} w_{1}^{*} u_{2} w_{2}^{*} t_{1}^{*} t_{2}^{*} \cap\left|m_{0}\right|$ fails to be context free and we are through.

Combining theorem 15 and 16 and corollary 13 and 14 we have the following result.

Theorem 17 There is a polynomial time algorithm to determine whether for a given equational Petri net $E$ there exists a marking $m_{0}$ such that $\left|m_{0}\right| T$ fails to be regular - or context free.

\subsection{The Equality Problem}

We now turn to the problem of determining whether two parallel systems over the same ranked alphabet $\Omega$ have the same behavior. In terms of equational Petri nets the problem in its simplest form can be phrased as follows: given two equational Petri nets with the same set of transitions $T$, decide whether the same firing sequences are enabled in the two nets. We will show that this problem can be answered in non-deterministic linear space. In fact, a slightly more general result holds.

Theorem 18 Given two $\Gamma$-labeled marked equational Petri nets $\left\langle E_{i} ; m_{i} ; h_{i}, \Gamma\right\rangle, i=1,2$, where $h_{2}$ is injective, the problem to decide whether $\mathcal{L}^{*}\left(m_{1} ; E_{1}\right) \subset \mathcal{L}^{*}\left(m_{2} ; E_{2}\right)$ is in PSPACE.

Proof. Let $\omega$ be an arbitrary symbol in $\Gamma$. For all positions $p$ in $P_{1}$ and all markings $m$ define

$$
c_{p}:=\max \left(i \leq \infty \mid \omega^{i} \in \mathcal{L}^{\infty}\left(p ; E_{1}\right) \cup \mathcal{L}^{*}\left(p ; E_{1}\right)\right)
$$

and

$$
\mu_{1}^{\omega}(m):=\Sigma_{p \in P_{1}} c_{p} m(p)
$$

Thus for a marking $m \mu_{1}^{\omega}(m)$ is the maximum number of transitions labeled $\omega$ that can be fired at $m$. Define $\mu_{2}^{\omega}$ similarly for the net $E_{2}$. For every symbol $\omega$ in $\Gamma$ we will determine a bound on the length of the shortest firing sequence $w \in \mathcal{F}^{*}\left(E_{1} ; m_{1}\right)$ - if such a sequence exists at all - that has following two properties:

$$
\begin{aligned}
& h_{1}(w) \in \mathcal{L}^{*}\left(m_{2} ; E_{2}\right) \\
& \mu_{1}^{\omega}\left(m_{1} \cdot w\right)>\mu_{2}^{\omega}\left(m_{2} \cdot h_{2}^{-1}\left(h_{1}(w)\right)\right) .
\end{aligned}
$$

As $\mathcal{L}^{*}\left(m_{1} ; E_{1}\right) \subset \mathcal{L}^{*}\left(m_{2} ; E_{2}\right)$ iff no such $\omega$ and $w$ exists this will prove our claim. We may safely assume that $\mathcal{L}^{*}\left(m_{1} ; E_{1}\right)$ lies completely in the range of $h_{2}$; otherwise one can easily construct a witness $x \in \mathcal{L}^{*}\left(m_{1} ; E_{1}\right)-\mathcal{L}^{*}\left(m_{2} ; E_{2}\right)$ of length at most $\mathrm{n}-1$. Hence $h_{2}^{-1}\left(h_{1}(w)\right)$ is defined for all $w \in\left|m_{1}\right| T$; we will write $g(w):=h_{2}^{-1}\left(h_{1}(w)\right)$. We will show that the desired bound is:

$$
B:=\gamma^{2 n+2} \cdot \max \left(r k\left(m_{1}\right), r k\left(m_{2}\right)\right)+n .
$$

Here $\gamma:=\max \left(\max \left(r k\left(\delta_{1}(t)\right) \mid t \in T_{1}\right), \max \left(r k\left(\delta_{2}(t)\right) \mid t \in T_{2}\right)\right)$ and $n:=\left|P_{1}\right|$. We may safely assume that $\gamma \geq 2$, otherwise both nets are essentially finite automata and our claim follows from standard 
results on regular languages. Taking the bound $B$ for granted, a PSPACE algorithm can be given as follows: non-deterministically generate a firing sequence $w$ in $T_{1}^{*}$ of length at most $B$ and verify that $w \in\left|m_{1}\right| T_{1}$ but $g(w) \notin\left|m_{2}\right| T_{2}$. The markings that appear during the firing of $w$ and $g(w)$ all have rank $O\left(\gamma^{\mathrm{en}}\right)$. In binary notation their size is therefore linear in the size of the input $\left(E_{1} ; m_{1}\right)$ and $\left\langle E_{2} ; m_{2}\right\rangle$. Hence the problem is solvable in non-deterministic linear space and thus in deterministic quadratic space by Savitch's theorem.

We now return to our main argument. A self-loop at a place $p$ is a transition $t$ enabled at $p$ such that $\Delta(t)(p) \geq 0$. Clearly a self-loop at $p$ labeled $\omega$ causes $c_{p}=\infty$. Let $q_{2}$ in $P_{2}$ be the place that enables a transition labeled $\omega$ in $E_{2}$. Note that by our assumption $q_{2}$ is uniquely determined and $\mu_{2}^{\omega}(m)=\infty$ or $\mu_{2}^{\omega}(m)=m\left(q_{2}\right)$ depending on whether $q_{2}$ has a self-loop labeled $\omega$ or not. Here a self-loop at place $p$ is understood to be a transition $t$ enabled at $p$ such that $\Delta(t)(p) \geq 0$. Now suppose $w$ is a minimal witness satisfying the above conditions 10 and 11 .

Case 1 The place $q_{2}$ has no self-loop labeled $\omega$ in $E_{2}$.

First notice that we can restrict ourselves to the case where there is no cycle of transitions in $E_{1}$ all labeled by $\omega$. For otherwise one can construct a witness $v$ of length at most $n-1$ such that $\mu_{1}^{\omega}\left(m_{1} \cdot v\right)=\infty>\mu_{2}^{\omega}\left(m_{2} \cdot g(v)\right)$ and there is nothing to show. Thus for any marking $m$ we have $\mu_{1}^{\omega}(m)<\infty$. Now let $\beta$ be the longest branch in $T R\left(m_{1}, w\right)$. By 12 the depth of $T R\left(m_{1}, w\right)$ is a least $n+2$, hence $\beta$ has length at least $n+2$. Let $z_{0}, z_{1}, \ldots, z_{n}$ be the $n+1$ bottom-most interior nodes on $\beta$ ( $z_{0}$ being the father of the leaf). Hence $30 \leq i<j \leq n\left(\lambda\left(z_{i}\right)=\lambda\left(z_{j}\right)\right.$ ) by the pigeonhole principle. Let $u$ be a firing sequence associated with the subtree of $z_{j}$ minus the subtree of $z_{i}$, and $w_{2}$ a firing sequence associated with the subtree of $z_{i}$. One can rearrange $w$ to a firing sequence $\bar{w}=w_{1} u w_{2} \in\left|m_{1}\right|$. By our choice of $u$ the firing sequence $w_{1} w_{2}$ is also enabled at $m_{1}$. But $\left|w_{1} w_{2}\right|<|\bar{w}|=|w|$, hence $g\left(w_{1} w_{2}\right)$ is enabled at $m_{2}$. Now $\mu_{1}^{\omega}\left(m_{1} \cdot \bar{w}\right)=\mu_{1}^{\omega}\left(m_{1} \cdot w\right)>\mu_{2}^{\omega}\left(m_{2} \cdot g(w)\right)=\mu_{2}^{\omega}\left(m_{2} \cdot g(\bar{w})\right)$ and $\mu_{1}^{\omega}\left(m_{1} \cdot \bar{w}\right)=\mu_{1}^{\omega}\left(m_{1} \cdot w_{1} w_{2}\right)+d_{1}, \mu_{2}^{\omega}\left(m_{2} \cdot g(\bar{w})\right)=\mu_{2}^{\omega}\left(m_{2} \cdot g\left(w_{1} w_{2}\right)+d_{2}\right.$ where $d_{1}:=\mu_{1}^{\omega}\left(\Delta_{1}(u)\right)$ and $d_{2}:=\mu_{2}^{\omega}\left(\Delta_{2}(g(u))\right)$ are the respective gains in net $E_{1}$ and $E_{2}$ during the firing of $u$ and $g(u)$. Note that $\Delta_{1}(u) \geq 0$, whence $d_{1} \geq 0$. Again by the minimality of $w$ we must have $\mu_{1}^{\omega}\left(m_{1} \cdot w_{1} w_{2}\right) \leq$ $\mu_{2}^{\omega}\left(m_{2} \cdot g\left(w_{1} w_{2}\right)\right)$. Thus $d_{1}>d_{2}$. Also observe that $|u| \leq \gamma^{n}$ by our choice of $z_{j}$. By deleting repetitious labels on $\beta$ above $z_{j}$ one can construct a firing sequence $\bar{u}$ enabled at $m_{1}$ of length at most $n-1$ such that $\bar{u} u^{*} \subset\left|m_{1}\right|_{T}$. But we have just seen that $d_{1}>d_{2}$, hence there exists a $k \leq r k\left(m_{2}\right)$ such that $g\left(\bar{u} u^{k}\right) \notin\left|m_{2}\right| r$ and we are done.

Case 2 The place $q_{0}$ has a self-loop labeled $\omega$ in $E_{2}$.

Note that in this case we must have $\mu_{1}^{\omega}\left(m_{1} \cdot w\right)>\mu_{2}^{\omega}\left(m_{2} \cdot w\right)=0$. Again let $\beta$ be the longest branch in $T R\left(m_{1}, w\right)$. By 12 let $z_{0}, \ldots, z_{2 n}$ be the $2 n+1$ bottom-most interior nodes on $\beta\left(z_{0}\right.$ being the father of the leaf). By the pigeonhole principle we have

$$
\exists 0 \leq i<j \leq r<s \leq 2 n\left(\lambda\left(z_{i}\right)=\lambda\left(z_{j}\right) \wedge \lambda\left(z_{r}\right)=\lambda\left(z_{s}\right)\right) .
$$

One can rearrange $w$ to a firing sequence $\bar{w}=w_{1} v w_{2} u w_{3} \in\left|m_{1}\right| r$ where $u$ is a firing sequence associated with the subtree of $z_{j}$ minus the subtree of $z_{i}, v$ is a firing sequence associated with the subtree of $z_{s}$ minus the subtree of $z_{r}$, and lastly $w_{3}$ a firing sequence associated with the subtree of $z_{i}$. As $w$ is a minimal witness we must have $g\left(w_{1} v w_{2} w_{3}\right) \in\left|m_{2}\right| T$. Hence the gain in the second net during the firing of $g(u)$ in position $q_{2}$ is not positive: $d_{2}:=\mu_{2}^{\omega}\left(\Delta_{2}(g(u))\right)=\mu_{2}^{\omega}\left(m_{2} \cdot g(w)\right)-$ $\mu_{2}^{\omega}\left(m_{2} \cdot g\left(w_{1} v w_{2} w_{3}\right)\right) \leq 0$. If $d_{2}<0$ one can use the argument from case 1 , so assume $d_{2}=0$. Then $\mu_{2}^{\omega}\left(m_{2} \cdot g\left(w_{1} v w_{2} w_{3}\right)\right)=0$ and by the minimality of $w \mu_{1}^{\omega}\left(m_{1} \cdot w_{1} v w_{2} w_{3}\right)=0$. It follows that $d_{1}:=\mu_{1}^{\omega}\left(\Delta_{1}(u)\right) \geq 1$. Now consider the firing sequence $w_{1} w_{2} u w_{3}$. Note that $\mu_{1}^{\omega}\left(m_{1} \cdot w_{1} w_{2} u w_{3}\right) \geq 1$. Hence, again by our choice of $w, g\left(w_{1} w_{2} u w_{3}\right)$ is enabled at $m_{2}$ and $\mu_{2}^{\omega}\left(m_{2}, g\left(w_{1} w_{2} u w_{3}\right)\right) \geq 1$. Thus $d_{2}^{\prime}:=\mu_{2}^{\omega}\left(\Delta_{2}(g(v))\right)=\mu_{2}^{\omega}\left(m_{2} \cdot g(w)\right)-\mu_{2}^{\omega}\left(m_{2} \cdot g\left(w_{1} w_{2} u w_{3}\right)\right)<0$ whereas $d_{1}^{\prime}:=\mu_{1}^{\omega}\left(\Delta_{1}(v)\right) \geq 0$. As in case 1 there is a firing sequence $\bar{v}$ of length at most $n-1$ such that $\bar{v} v^{*} \subset\left|m_{1}\right|_{T}$. However, for some $k \leq r k\left(m_{2}\right)+n \cdot B, g\left(\tilde{v} v^{k}\right) \notin \mathcal{L}^{*}\left(E_{2} ; m_{2}\right)$. This completes the proof. 
Corollary 19 Given two marked $E P N s\left\langle E_{i} ; m_{i}\right\rangle, i=1,2$, with free labelings $h_{i}: T_{i} \rightarrow \Sigma$ the Equality Problem as well as the Subset Problem are in PSPACE. I.e., there are algorithms with polynomial space complexity to decide whether $\mathcal{L}^{*}\left(m_{1} ; E_{1}\right)=\mathcal{L}^{*}\left(m_{2} ; E_{2}\right)$ and whether $\mathcal{L}^{*}\left(m_{1} ; E_{1}\right) \subset \mathcal{L}^{*}\left(m_{2} ; E_{2}\right)$.

We conclude by stating some open problems. Corollary establishes an upper bound for the Equality as well as the Subset Problem in equational Petri nets with free labelings. We are not aware of any lower bounds. Furthermore, it would be interesting to know whether these problems are decidable for equational Petri nets that satisfy the disjoint labeling condition and ultimately for arbitrarily labeled nets.

\section{BIBLIOGRAPHY}

[1] M. Hack. The equality problem for vector addition systems is undecidable. Theoretical Computer Science, $2: 77-95,1976$.

[2] R.R. Howell, L.E. Rosier, and H. C. Yen. An O( $\left.n^{1.5}\right)$ algorithm to decide boundedness for conflict free vector replacement systems. Technical report, University of Texas at Austin, 1986.

[3] D.T. Huynh. Commutative grammars: the complexity of the uniform word problems. Information and Control, 57:21-39, 1983.

[4] M. Jantzen. Complexity of place and transition systems. In Petri Nets: Central Models and their Properties, volume 254 of LNCS. Springer-Verlag, 1986.

[5] M. Jantzen. Language theory of Petri nets. In Petri Nets: Central Models and their Properties, volume 254 of LNCS. Springer-Verlag, 1986.

[6] W. Reisig. Petri Nets, volume 4 of EATCS. Springer Verlag, 1986.

[7] G. Rozenberg and R. Verraedt. Subset languages of Petri nets part I: The relationship to string languages and normal forms. Theoretical Computer Science, 26:301 - 326, 1983.

[8] G. Rozenberg and R. Verraedt. Subset languages of Petri nets part II: The closure properties. Theoretical Computer Science, 27:85-108, 1983.

[9] R.Valk and G.Vidal-Naquet. Petri nets and regular languages. Journal of Computers and System Science, $23: 299-325,1981$. 